\title{
Dose assessment by quantification of chromosome aberrations and micronuclei in peripheral blood lymphocytes from patients exposed to gamma radiation
}

\author{
Isvânia Silva-Barbosa ${ }^{1}$, Símey Pereira-Magnata ${ }^{1}$, Ademir Amaral $^{1}$, Graça Sotero ${ }^{2}$ and \\ Homero Cavalcanti Melo ${ }^{3}$ \\ ${ }^{1}$ Universidade Federal de Pernambuco, Departamento de Energia Nuclear, Grupo de Estudos em \\ Radioproteção e Radioecologia (GERAR), Recife, PE, Brazil. \\ ${ }^{2}$ Fundação de Hematologia e Hemoterapia de Pernambuco, Recife, PE, Brazil. \\ ${ }^{3}$ Hospital do Câncer, Centro de Radioterapia de Pernambuco, Recife, PE, Brazil.
}

\begin{abstract}
Scoring of unstable chromosome aberrations (dicentrics, rings and fragments) and micronuclei in circulating lymphocytes are the most extensively studied biological means for estimating individual exposure to ionizing radiation (IR), which can be used as complementary methods to physical dosimetry or when the latter cannot be performed. In this work, the quantification of the frequencies of chromosome aberrations and micronuclei were carried out based on cytogenetic analyses of peripheral blood samples from 5 patients with cervical uterine cancer following radiotherapy in order to evaluate the absorbed dose as a result of partial-body exposure to ${ }^{60} \mathrm{Co}$ source. Blood samples were collected from each patient in three phases of the treatment: before irradiation, $24 \mathrm{~h}$ after receiving $0.08 \mathrm{~Gy}$ and $1.8 \mathrm{~Gy}$, respectively. The results presented in this report emphasize biological dosimetry, employing the quantification of chromosome aberrations and micronuclei in lymphocytes from peripheral blood, as an important methodology of dose assessment for either whole or partial-body exposure to IR.
\end{abstract}

Key words: chromosome aberrations, micronuclei, biodosimetry, partial-body irradiation, lymphocyte.

Received: August 13, 2004; Accepted: January 22, 2005.

\section{Introduction}

Absorbed dose is the most important physical quantity for evaluating potential biological response as a result of exposure to ionizing radiation (IR). Physical dosimetry is commonly performed by instruments that are sensitive to the physical effects of IR. However, in most cases involving real or suspected accidental exposure, people are not wearing a dosimeter and, as a result, physical dosimetry is not straightforward. For such situations, the study of early biological effects induced by an exposure to IR has been proposed as either a complementary or an alternative method for dose assessment (Downing, 2000; Amaral, 2002; Bonassi and $\mathrm{Au}, 2002$; Ramalho and Nascimento, 1991; Ramalho et al., 1995; Voisin et al., 2001).

Biological dosimetry (biodosimetry) is based on the investigation of radioinduced biological effects (bioindicators) in order to correlate them with the radiation dose. Among the bioindicators employed in biodosimetry,

Send correspondence to Isvânia Maria Serafim Silva-Barbosa. Universidade Federal de Pernambuco, Departamento de Energia Nuclear, Av. Prof. Luiz Freire 1000, 50740-540 Recife, PE, Brazil. E-mail: isvania@uol.com.br. the scoring of chromosome aberrations (CA) is the most reliable method for evaluating individual exposure (Du Frain et al., 1980; Bender et al., 1988; Voisin, 1997; Albertini et al., 2000). Scoring of radio-induced CA from peripheral blood lymphocytes has been developed into a valuable dosimetric tool in radiological protection (IAEA, 2001). In particular, unstable CA (dicentrics, rings and fragments) are generally considered as specific to radiation exposure, and these types of aberrations are referred to as unstable CA because of their persistence in the body decline with cell division cycles (Amaral, 2002).

On the other hand, micronuclei (MN), a kind of unstable CA byproduct, are cytoplasm chromatin masses that arise from centric or acentric products of damaged chromosomes. They have the appearance of small nuclei, in addition to the cell's nucleus (Köksal et al., 1996; Uma Devi et al., 1998; IAEA, 2001), and they are identified during the division of mitogen-activated human lymphocytes by blocking cytokinesis (Uma Devi et al., 1998). As the scoring of $\mathrm{MN}$ is more sensitive and faster than the scoring of $\mathrm{CA}$, improvements in MN methodology for biodosimetry have been tested (IAEA, 2001). 
For dose assessment purposes, the choice of the circulating lymphocytes in human blood is adequate because samples are easily obtained from the peripheral blood and the vast majority of peripheral lymphocytes reside in the $\mathrm{G}_{0}$ phase of the cell cycle, representing a cell population which is predominantly in a DNA presynthetic stage. When mitogen stimulation converts these resting lymphocytes into cycling cells, DNA lesions can be visualized in the metaphase chromosome (Lindholm, 2000).

Biological dosimetry using the analysis of unstable chromosomal aberrations in human lymphocytes from peripheral blood is well established and accurate, especially in the case of acute exposure, and when the blood samples are obtained within few days after the real or suspected radiation exposure (Lucas, 1997; IAEA, 2001; Camparoto et $a l ., 2003)$.

As in most cases of accidents involving exposure to ionizing radiation concern partial-body irradiation, the goal of this work was to evaluate the scoring of the frequency of $\mathrm{CA}$ and $\mathrm{MN}$, in circulating lymphocytes, for dose evaluation in such situations. Thus, this study was carried out based on cytogenetic analyses of peripheral blood samples from patients with cervical uterine cancer following treatment based on partial-body irradiation with ${ }^{60} \mathrm{Co}$.

\section{Material and Methods}

\section{Patients}

The studied group was composed of five women under treatment for cervical uterine cancer at the Radiotherapy Department of the Hospital do Câncer (Recife, Brazil). All patients (ages varying between 40 and 59) provided written informed consent before the beginning of this study, for approval from the ethical practices committee of that Hospital. None of the studied individuals had been exposed to IR in the two years before the radiotherapy.

\section{Radiotherapy and dosimetry}

No changes in the hospital's protocol for patient treatment were made because of this research. Details such as dose and dose-rate delivered to the patients were provided by the physician responsible for the treatment in that hospital. The same protocol for radiotherapy was employed for all patients included in this study. Primarily, 0.08 Gy was delivered to the patient's irradiated volume during the phase of tumor localization. Henceforth, for each radiotherapy session, a mean dose of 1.8 Gy was delivered to the irradiated volume of the patient, using a source of ${ }^{60} \mathrm{Co}$ (dose-rate: $0.33 \mathrm{cGy} \cdot \mathrm{min}^{-1}$ ).

\section{Lymphocyte sampling}

The patient's blood samples were obtained in sterile heparinized tubes. $5 \mathrm{~mL}$ of peripheral blood were collected prior to any medical radiation exposure. Similarly, equal volumes of blood samples were collected $24 \mathrm{~h}$ after tumor localization and the first radiotherapy session, respectively. From each patient, blood samples were obtained in three different situations, corresponding to the absorbed doses of 0.0 (background radiation), 0.08 and $1.8 \mathrm{~Gy}$. The samples were transported to the cytogenetic laboratory (HEMOPE Recife, Brazil), in an ice bath, according to IAEA recommendations (2001). Although the protocol included additional radiotherapy sessions, in the present work only the three cited phases of the treatment were taken into account.

\section{Culture set up for CA analyses}

For the chromosome aberration studies, $0.3 \mathrm{~mL}$ of the blood samples were cultured for $48 \mathrm{~h}$, in a humidified atmosphere containing $5 \% \mathrm{CO}_{2}$, at $37{ }^{\circ} \mathrm{C}$. The culture medium consisted of $4 \mathrm{~mL}$ of RPMI-1640 (Sigma-Aldrich, Irvine, UK) supplemented with $20 \%$ fetal bovine serum. Besides this, $0.1 \mathrm{~mL}$ of phytohemagglutinin (PHA-Gibco BRL, Grand Island, NY) was added to stimulate cell division. To block the mitotic process of the cells at the metaphase stage, Colcemid (Gibco BRL) was added for the last $2 \mathrm{~h}$ of culture at a final concentration of $0.1 \mu \mathrm{g} / \mathrm{mL}$. The cells were harvested, given hypotonic shock treatment with $75 \mathrm{mM} \mathrm{KCl}$ and fixation with methanol-acetic acid (ratio 1:4 v/v). Slides were prepared for each sample and stained with $10 \%$ Giemsa (Merk) and mounted. The number of aberrations was observed under an Olympus microscope, using the 100x objective, and a cell was considered as aberrant if it had one or more unstable CA (dicentric, rings and chromosome fragments).

\section{Culture set up for MN analyses}

The presence of MN in a binucleated cell was assayed by blocking the cell at the cytokinesis stage as described by Fenech and Morely (1985). Peripheral blood lymphocytes were cultured for $72 \mathrm{~h}$, in a humidified atmosphere containing $5 \% \mathrm{CO}_{2}$, at $37^{\circ} \mathrm{C}$; to this, a blood sample of $0.3 \mathrm{~mL}$ was added to the culture medium, which consisted of $4 \mathrm{~mL}$ of RPMI-1640 (Sigma-Aldrich, Irvine, UK) supplemented with $20 \%$ fetal bovine serum. Besides this, $0.1 \mathrm{~mL}$ phytohemagglutinin (PHA-Gibco BRL, Grand Island, NY) was added to stimulate cell division. To block the cells at the telophase stage, citocalasine-B was added for the last 24 $\mathrm{h}$ of culture at a final concentration of $5 \mu \mathrm{g} / \mathrm{mL}$. Harvesting involved isotonic shock with $\mathrm{NaCl}(0.9 \%)$ and fixation with acetic acid (ratio 1:4 v/v), slides were prepared for each sample and stained with $10 \%$ Giemsa (Merk). The investigation into the number of $\mathrm{MN}$ was observed under an Olympus microscope using the 100x objective.

\section{Results and Discussion}

\section{Chromosome aberrations}

Table 1 presents the number of unstable CA (dicentrics and fragments) in lymphocytes, as well as their 
Table 1 - Distribution of unstable CA (dicentrics and fragments) for absorbed doses of 0 Gy (background), 0.08 Gy and 1.8 Gy.

\begin{tabular}{lccccccc}
\hline Dose (Gy) & Patient (age) & CS & NCA & ND & NF & FD & FCA \\
\hline 0 & A (40) & 1000 & 1 & 1 & 0 & 0.0010 & 0.0010 \\
& B (42) & 1000 & 1 & 1 & 0 & 0.0010 & 0.0010 \\
C (44) & 1150 & 2 & 1 & 1 & 0.0009 & 0.0017 \\
D (53) & 1000 & 1 & 1 & 0 & 0.0010 & 0.0010 \\
E (59) & 1200 & 2 & 2 & 0 & 0.0017 & 0.0017 \\
\hline Mean \pm SD & & & & & $0.0011 \pm 0.0003$ & $0.0013 \pm 0.0004$ \\
\hline A (40) & 450 & 1 & 1 & 0 & 0.0022 & 0.0022 \\
B (42) & 610 & 1 & 1 & 0 & 0.0016 & 0.0016 \\
C (44) & 550 & 1 & 1 & 0 & 0.0018 & 0.0018 \\
D (53) & 501 & 1 & 1 & 0 & 0.0020 & 0.0020 \\
E (59) & 820 & 2 & 1 & 1 & 0.0012 & 0.0024 \\
Mean \pm SD & & & & & $0.0018 \pm 0.0004$ & $0.0020 \pm 0.0003$ \\
\hline A (40) & 280 & 11 & 4 & 7 & 0.0143 & 0.0393 \\
B (42) & 282 & 11 & 5 & 6 & 0.0177 & 0.0390 \\
C (44) & 214 & 8 & 3 & 5 & 0.0140 & 0.0374 \\
D (53) & 200 & 9 & 4 & 5 & 0.0200 & 0.0450 \\
E (59) & 260 & 10 & & 6 & 0.0154 & 0.0385 \\
Mean \pm SD & & & & & $0.0163 \pm 0.0025$ & $0.0398 \pm 0.0030$ \\
\hline
\end{tabular}

CS - cell scored; NCA - number of unstable chromosome aberrations; ND - number of dicentrics; NF - number of fragments; FD - frequency of dicentrics; FCA - frequency of unstable aberrations.

frequencies for all patients, before radiation exposure, and after receiving doses of 0.08 and $1.8 \mathrm{~Gy}$, respectively.

In the case of the 0 and $0.08 \mathrm{~Gy}$, the number of fragments was negligible, and the results concerning the frequency of dicentrics and the frequency of total unstable CA were obviously the same. Fragments were observed as a result of the exposure to the dose of the $1.8 \mathrm{~Gy}$, which justified the difference between dicentric and total CA frequency.

For evaluating the mean absorbed dose in the irradiated volume based on these results, Eq. (1) was employed, which was experimentally obtained by Lloyd et al. (1986), by following in vitro irradiation of blood samples and using the protocol suggested by the International Atomic Energy Agency (IAEA, 1986). In this study the authors investigated whole-body exposures through the scoring of the frequency of unstable CA (dicentrics, rings and chromosome fragments) by in vitro blood irradiation with ${ }^{60} \mathrm{Co}$ (dose-rate 0.5 Gy. $\mathrm{min}^{-1}$ ) of fresh human blood from a panel of healthy volunteers, obtaining the following calibration curve:

$$
Y=0.001+0.0164 D+0.0493 D^{2}
$$

In the above equation $Y$ represents the frequency of unstable CA (dicentrics and fragments) scored after irradiation as a function of the dose $D$ in grays (Gy).

To relate the frequency of CA obtained experimentally with those predicted by Eq. (1), one must considerer that $24 \mathrm{~h}$ after partial-body exposure to ${ }^{60} \mathrm{Co}$, the irradiated lymphocytes will be redistributed in peripheral blood, since the organ-circulating blood turnover takes about $30 \mathrm{~min}$ (IAEA, 2001). Next, taking into account a homogeneous distribution of blood in the human body (Milnor, 1974), it can be inferred that $24 \mathrm{~h}$ after irradiation the frequency of unstable chromosome aberrations in peripheral blood will be proportional to the percentage of the patient's irradiated volume. According to the data from the planning of dose distributions, the irradiated volume of each patient corresponded to about $1 / 5$ of the total volume of their bodies.

Based on the above remarks, one can compare the frequencies of CA obtained experimentally and those predicted directly by substituting the dose value in Eq. (1). However, as the frequency of CA obtained from blood samples is a result of a partial-body exposure, the values shown in Table 1 for the frequency of CA (FCA) must be multiplied by 5 to correlate it with a whole-body exposure for it to be in agreement with the frequency of CA predicted by Eq. (1). Obviously, this reasoning is not applied to spontaneous aberrations (background) that depend only on natural radiation. Table 2 summarizes this comparison.

For the dose of $0.08 \mathrm{~Gy}$, there is a great difference between the frequencies experimentally obtained and those predicted by Eq. (1). The relationship between the accuracy of this method is strongly dependent on the number of metaphases scored. For example, a detection limit of about 
Table 2 - Comparison between estimated and experimental frequencies of unstable CA.

\begin{tabular}{lcc}
\hline Dose $(\mathrm{Gy})$ & $\bar{Y}$ (predicted by Eq. (1)) & $Y$ (this work*) \\
\hline Background & 0.0010 & 0.0013 \\
0.08 & 0.0026 & 0.0100 \\
1.8 & 0.1900 & 0.1990 \\
\hline
\end{tabular}

*After considering the irradiated volume of the body.

$0.2 \mathrm{~Gy}$ is related to the analysis of 500 metaphases (Voisin, 1997), which is the case here.

\section{Micronuclei}

Table 3 shows the scored binucleated cells (SBC) and micronuclei (SMN), as well as the frequency of MN (FMN) in lymphocytes for the same blood samples. FMN is expressed as a percentage ratio of the total number of MN to the observed binucleated cells.

To investigate whole body exposures, Voisin et al. (2001) studied the frequency of $\mathrm{MN}$ by in vitro blood irradiation with ${ }^{60} \mathrm{Co}$ (dose-rate $0.5 \mathrm{~Gy} \cdot \mathrm{min}^{-1}$ ) of peripheral blood samples from 47 healthy individuals (ages varying from 25 and 30), obtaining the following calibration curve:

$$
Y=0.012+0.071 D+0.028 D^{2}
$$

Here, $Y$ represents the frequency of $\mathrm{MN}$ noted after irradiation as a function of the dose $D$, in grays (Gy).

Table 3 - Distribution of the frequencies of micronuclei for the absorbed doses of 0 Gy (background), 0.08 Gy and $1.8 \mathrm{~Gy}$.

\begin{tabular}{|c|c|c|c|c|}
\hline Dose (Gy) & Patient (age) & $\mathrm{SBC}^{*}$ & $\mathrm{SMN}^{*}$ & FMN* \\
\hline \multirow[t]{6}{*}{0} & A (40) & 1200 & 01 & 0.0008 \\
\hline & B (42) & 1140 & 01 & 0.0009 \\
\hline & C (44) & 1500 & 02 & 0.0013 \\
\hline & $\mathrm{D}(53)$ & 1000 & 01 & 0.0010 \\
\hline & $\mathrm{E}(59)$ & 1820 & 03 & 0.0016 \\
\hline & Mean \pm SD & & & $0.0011 \pm 0.0003$ \\
\hline \multirow[t]{6}{*}{0.08} & A (40) & 1415 & 03 & 0.0021 \\
\hline & B (42) & 1000 & 02 & 0.0020 \\
\hline & $\mathrm{C}(44)$ & 1500 & 03 & 0.0020 \\
\hline & $\mathrm{D}(53)$ & 1400 & 03 & 0.0021 \\
\hline & E (59) & 1640 & 04 & 0.0024 \\
\hline & Mean \pm SD & & & $0.0021 \pm 0.0002$ \\
\hline \multirow[t]{6}{*}{1.8} & $\mathrm{~A}(40)$ & 1000 & 41 & 0.0410 \\
\hline & B (42) & 1000 & 52 & 0.0520 \\
\hline & $\mathrm{C}(44)$ & 450 & 21 & 0.0477 \\
\hline & $\mathrm{D}(53)$ & 1000 & 55 & 0.0550 \\
\hline & $\mathrm{E}(59)$ & 1200 & 79 & 0.0658 \\
\hline & Mean \pm SD & & & $0.0523 \pm 0.0092$ \\
\hline
\end{tabular}

*SBC - Scored Binucleated Cells; SMN - Scored Micronuclei; FMN Frequency of Micronuclei.
Using Eq. (2) to predict the frequency of $\mathrm{MN}$, one obtains 0.018 and 0.230 respectively for the absorbed doses of 0.08 and 1.8 Gy (Table 4). In order to compare the predicted frequencies with the ones shown in Table 3, one must remember that all patients were partially exposed to IR. As for chromosome aberrations, $24 \mathrm{~h}$ after irradiation the exposed lymphocytes are expected to be homogenously distributed in the patient's body. As a result, the frequency of $\mathrm{MN}$ is therefore about $1 / 5$ of the value predicted by Eq. (2). Thus, to compare the frequency of MN experimentally obtained with those predicted from Eq. (2), one should multiply the experimental values by 5 , following the same reasoning as for chromosome aberrations. The results to dose of the 1.8 Gy are in good agreement with each other as summarized in Table 4.

Observing the background level (0.012) in Eq. (2), one may infer that in the group used to determine the equation the spontaneous frequency of $\mathrm{MN}$ is higher than the similar frequency obtained from the group investigated is this work (0.0011). This may justify the great difference observed between the values presented for the expected frequencies of $\mathrm{MN}$ and those obtained in this work. On the other hand, disregarding the background level in this equation to predict the frequency of $\mathrm{MN}$, for dose of 0.08 Gy, yields a result of 0.006 , which is in better agreement with the experimental value of 0.010 (Table 4) than the analyses by chromosomal aberration. Indeed, several works have shown that the detection limit of the methodology based on scoring of MN is lower than that of chromosome aberrations (Voisin, 1997; Voisin et al., 1999; IAEA, 2001).

This work was designed to investigate the use of both methodologies (scoring of CA and MN) for dose evaluation from a simulation of a partial-body exposure to IR. This was possible due to the characteristics of treatment and health conditions of each subject. Although all patients presented a diagnosis of cervical cancer, the reason for choosing them was judicious because their general health aspects were appropriate for the objective of this research. The normal leucogram test was the basic parameter for inclusion of each of the 5 patients. For all patients, the results obtained for spontaneous chromosome aberrations and micronuclei (Tables 1 and 3), corresponding to an individual exposure to background radiation, are in good agreement with those presented in literature. This contributes to the fact that those

Table 4 - Comparison between the values expected by Eq. (2) and the frequency of micronuclei obtained in this research, considering partial-body exposure.

\begin{tabular}{lcc}
\hline Dose $(\mathrm{Gy})$ & $\bar{Y}$ (predicted by Eq. (2)) & $Y$ (this work*) \\
\hline Background & 0.012 & 0.0011 \\
0.08 & 0.018 & 0.010 \\
1.8 & 0.230 & 0.262 \\
\hline
\end{tabular}

*After considering the irradiated volume of the body. 
patients can be considered healthy individuals that were partially exposed to ionizing radiation.

To interpret the scoring of either $\mathrm{CA}$ or $\mathrm{MN}$ in terms of radiation dose, a calibration curve (frequency of CA or $\mathrm{MN}$ versus dose) is necessary. For this interpretation, calibration curves obtained in vivo and in vitro were employed. In fact, since the radiosensitivity of lymphocytes in vitro and in vivo are the same, the dose-effect relationship obtained after in vitro irradiation of blood (as in the case of Eq. (1)) can therefore be used to estimate effects from an irradiation in vitro (IAEA, 2001).

Comparing the two methods in terms of material, similar material is employed in both, differing only in the time of cell culture and in the application of the citocalasine-B. Therefore, the requirements to implement one or the other are almost identical. In terms of workload, the MN method is faster than chromosome aberrations. For example, in conducting this work, the average number of metaphases observed per day was about 150 compared to 800 binucleated cells.

In this context, scoring of $\mathrm{CA}$ and $\mathrm{MN}$ can be extremely useful in the immediate response to accidents, where initial information is often confusing or scarce. For example, Lloyd and co-workers suggest an approach in which only 20 and 50 cells need to be scored initially, for medical triage of whole body and partial-body irradiation, respectively (Lloyd et al., 2000). Based on this approach one can reduce the counting workload.

Both methods reported in the present work can be improved with the employment of the Fluorescent In situ Hybridization (FISH) technique, which allows marked nucleic acid probes to be visualized on target tissues, cells, nuclei, and chromosomes so accurately that specific nuclei acid sequences can be located in the biological structure observed. On the basis of the FISH technique, using a pancentromeric probe, the observation of dicentrics is an indisputable advantage in terms of speed and simplicity compared to the drawbacks of the conventional "G banding" technique. However, FISH methodology remains an expensive technique and it is not essential in terms of medical-legal evaluation, where the length of time spent for scoring of either CA or MN does not represent a crucial parameter, as in the case of expertise in radiation protection.

\section{Conclusion}

In response to radiation accidents, dose estimates are crucial for risk assessment, as well as for clinical planning of treatment of highly exposed victims. In many accidents initial information generally becomes available only after detailed, and ambiguous, dose reconstruction. The results presented in this work point out that the scoring of either chromosome aberration or micronuclei are reliable methodologies for investigating exposure to ionizing irradiation, such as partial-body ones. However, according to the methodology presented and discussed here, more knowledge about the irradiated part of the individual is necessary. Apart from precise dose reconstructions, biodosimetry can also be used in the immediate response to accidents, where few cells need to be scored initially, and for medical triage of whole body and partial-body irradiation, respectively. In this case, this would play an important role in national emergency responses to a large-scale accident where many persons may have been exposed.

\section{Acknowledgments}

The authors are grateful to the Fundação Cooperação de Aperfeiçoamento de Pessoal de Nível Superior (CAPES, Brazil) and Conselho Nacional de Desenvolvimento Científico e Tecnológico (CNPq, Brazil) for supporting this work, and to Dr. Ernesto Roester together with the staff from Hospital do Câncer (Recife, PE, Brazil).

This work was performed in collaboration with the Fundação de Hematologia e Hemoterapia de Pernambuco (HEMOPE, Recife, PE, Brazil), in the cytogenetics laboratory under the supervision of Dr. Raul Melo.

\section{References}

Albertini RJ, Anderson D, Douglas GR, Hagmar L, Hemmink K, Merlo F, Natarajan AT, Norppa H, Shuker DE, Tice RR, Waters MD and Aitio A (2000) IPCS guidelines for the monitoring of genotoxic effects of carcinogens in humans. International program on chemical safety. Mutat Res 463:111-172.

Amaral A (2002) Trends in biological dosimetry: An overview. Braz Arch Biol Technol 45:119-124.

Bender MA, Awa AA, Brooks AL, Evans HJ, Groer PG, Littlefield LG, Pereira C, Preston RJ and Wachholz BW (1988) Current status of cytogenetic procedures to detect and quantify previous exposure to radiation. Mutat Res 196:103-159.

Bonassi S and Au WW (2002) Biomarkers in molecular epidemiology studies for health risk prediction. Mutat Res 511:73-86.

Camparoto ML, Ramalho AT, Natarajan AT, Curado MP and Sakamoto-Hojo ET (2003) Translocation analysis by the FISH-painting method for retrospective dose reconstruction in individuals exposed to ionizing radiation 10 years after exposure. Mutat Res 530:1-7.

Downing GJ (2000) Biomarkers and surrogate endpoints in clinical research: Definitions and conceptual model. In: Downing GJ (ed) Biomarkers and Surrogate Endpoints: Clinical Research and Applications. Elsevier, Amsterdam, pp 1-9.

Du Frain RJ, Littlefield LG, Joiner EE and Frome EL (1980) In vitro human cytogenetic dose-response systems. In: Hubner KF and Fry SA (eds) The Medical Basis for Radiation Accident Preparedness, Elsevier North-Holland, New York, pp 357-374.

Fenech M and Morley AA (1985) Measurement of micronucle in lymphocytes. Mutat Res 147:29-36.

IAEA - International Atomic Energy Agency (1986) Biological Dosimetry Chromosomal Aberration in Analysis for Dose Assessment. Publication n. 260, Vienna. 
IAEA - International Atomic Energy Agency (2001) Cytogenetic Analysis for Radiation Dose Assessment. Technical Report Series n. 405, Vienna.

Köksal G, Dalci DÖ and Pala FS (1996) Micronuclei in human lymphocytes: The Co-60 gamma-ray dose-response. Mutat Res 359:151-157.

Lindholm C (2000) Stable chromosome aberrations in the reconstruction of radiation doses. Radiation and Nuclear Safety Authority, STUK-A176, Helsinki pp 91.

Lloyd DC, Edwards AA and Prosser JS (1986) Chromosome aberrations induced in human lymphocytes by in vitro acute $\mathrm{X}$ and gamma radiation. Radiat Prot Dosim 15:83-88.

Lloyd DC, Edwards AA, Moquet JE and Guerero-Carbajal YC (2000) The role of cytogenetics in early triage of radiation causalities. Appl Rad Isot 52:1107-1112.

Lucas JN (1997) Dose reconstruction for individuals exposed to ionizing radiation using chromosome painting. Radiat Res 148:33-38.

Milnor WR (1974) In: Medical Physiology. 13th ed. Mount Castle, Mosby, Saint Louis, pp 920.
Ramalho AT and Nascimento ACH (1991) The fate of chromosomal aberrations in ${ }^{137} \mathrm{Cs}$-exposed individuals in the Goiania radiation accident. Health Phys 60:67-70.

Ramalho AT, Curado MP and Natarajan AT (1995) Lifespan of human lymphocytes estimated during a six year cytogenetic follow-up of individuals accidentally exposed in the 1987 radiological accident in Brazil. Mutat Res 331:47-54.

Uma Devi P, Satish Rao BS and Kamath R (1998) A method to score micronuclei in vivo using cytochalasin B-induced cytokinesis block. Mutat Res 401:33-37.

Voisin P (1997) Chromosome lesions as short and medium term biological indictor of acute irradiation. In: Court $\mathrm{L}$ and Lallemand A (eds) l'Homme Blessé. EDF, Paris, 357 pp.

Voisin P, Paillole N, Benderitter M, Claraz M, Chambrette V, Claraz M, Delbos M, Durand V, Leroy A and SorokineDurm I (1999) De l'expertise à la crise: Dicentriques ou micronoyaux. Dos Bio à la SARAM 15:24-28.

Voisin P, Benderitter M, Claraz M, Chambrette V, SorokineDurm I, Delbos M, Durand V, Leroy A and Paillole N (2001) The cytogenetic dosimetry of recent accidental overexposure. Cell Mol Bio 47:557-564.

Associate Editor: Catarina S. Takahashi 